Address correspondence to Dr Jeffrey S. Kutcher, Department of Neurology, Michigan House, 2301 Commonwealth Blvd., Room 1031, Ann Arbor, MI 48109,jkutcher@umich.edu. Relationship Disclosure: Dr Kutcher serves as a consultant for ElMindA, Ltd. and the National Hockey League Players Association and as director of the National Basketball Association concussion program. $\mathrm{He}$ is the principal investigator on a grant his institution receives from ElMindA, Ltd. Dr Giza has served as a consultant for Alcobra Pharma and Pearson PLC and as a consultant on patient referrals for Major League Soccer, the National Football League, and the National Hockey League Players Association; he serves on committees of Major League Soccer and the National Collegiate Athletic Association and on the data safety monitoring board of LA Biomed. Dr Giza serves as a speaker for the Medical Education Speakers Network; has given expert testimony on medicolegal cases; receives book royalties from Blackwell Publishing; receives payment for the development of educational presentations from American Physician Institute, the AAN, and the University of California, Los Angeles; and received travel and/or accommodations and/or meeting expenses from the AAN, California State Athletic Commission, Major League

Soccer, and the National Collegiate Athletic Association.

Dr Giza's institution receives the following in association with his work: research grant for a clinical project on traumatic brain injury from the Defense Advanced Research Projects Agency; a research grant on youth concussions from the Joseph Drown Foundation; research grants for collegiate concussions from the National Collegiate Athletic Association and the US Department of Defense; research grants for preclinical and clinical projects on traumatic brain injury from the NIH; and funding for the University of California, Los Angeles, Steve Tisch

\title{
Sports Concussion Diagnosis and Management
}

\author{
Jeffrey S. Kutcher, MD; Christopher C. Giza, MD
}

\begin{abstract}
Purpose of Review: To provide the neurologist with a framework for the clinical approach to sports concussion diagnosis and management.

Recent Findings: As the issue of brain injury in athletes has emerged and developed, shifting the landscape of public concern, neurologists have become more directly involved in the diagnosis and management of sports concussion. Neurologists are now playing an increased role in acute concussion diagnosis, early injury management, return-to-play decisions, and evaluation for potential long-term effects from exposure to biomechanical forces on brain health. Concussion is only one part of this spectrum, but it is no small concern. Sports concussion diagnosis and management require a comprehensive neurologic approach as the return-to-play decision is a medical one covering a spectrum of potential complications and future risks. Understanding the clinical syndrome of concussion as well as the underlying pathophysiologic mechanism is essential to providing care. Employing classic neurologic diagnostic techniques while concurrently respecting the unique nature of caring for athletes is also critical. Without an objective method of measuring the underlying metabolic injury, concussion management is, by necessity, a clinically intense endeavor that requires a broad skill set. Summary: Providing recommendations regarding the long-term effects of brain trauma and the need for retirement from contact sports requires an appreciation for both the reason for concern and the lack of data to frame this risk. As science continues to advance in this area, so will our diagnostic approaches and management schema. Neurologists caring for athletes with brain trauma should continue to seek the best possible evidence to help shape their clinical decisions.
\end{abstract}

Continuum (Minneap Minn) 2014;20(6):1552-1569.

\section{INTRODUCTION}

The field of neurology has long been involved in the diagnosis of cerebral concussion. In the early part of the 20th century, Joseph Babinski took a particular interest in the varying clinical effects produced from the blast-induced concussive injuries commonly seen in World War I soldiers. ${ }^{1}$ Later in the century, Derek Denny-Brown pondered the potential pathophysiologic constructs of concussion, publishing his work even while some of his contemporaries argued against the injury's very existence. ${ }^{2,3}$ In this early era of concussion neurology, no clear diagnostic schema or approach to management dominated. Neurologists knew only what they saw in their patients: a transient symptom complex demonstrating potential disturbances across the entire spectrum of brain function with no obvious effect on tissue or measurable physiology and no apparent long-term consequences. This compelling heterogeneity of concussion presentations, combined with the potential overlap with other known intracranial 
pathologic mechanisms and the lack of an objective test for the injury, fed neurology's rich early history of professional discourse on concussion.

Although many aspects of concussion were widely debated at the time, it was generally accepted that concussion was not a serious injury. In fact, the clinical relevance of concussion was debated throughout the remainder of the 20th century, even as the specific circumstance of concussion in athletes and military personnel began to evolve from a minor concern to a major public health issue. Given the unique exposure to impact forces experienced by contact sport athletes and the presumption of risk involved with participation in their sports, clinicians and scientists began to increase research efforts in the latter portion of the 20th century to bring much-needed clarity and understanding to the fore.

In 1997, the American Academy of Neurology (AAN) published its first practice parameter document on sports concussion, hoping to provide a clear framework for clinicians to diagnose and manage the injury. ${ }^{4}$ Specific concerns regarding potentially devastating consequences of the so-called second impact syndrome led to further worry about the amount of risk experienced by contact sport athletes, ushering in an era of increased injury surveillance and more conservative injury management. ${ }^{5,6}$ Further heightening public concern was the emerging idea of potential long-term brain health sequelae stemming from exposure to repetitive biomechanical forces. ${ }^{7,8}$ One of these potential conditions, chronic traumatic encephalopathy (CTE), is now a well-discussed, if not well-understood, diagnosis.

As the growing concern for these issues far outpaced the published science, a clear need for clinical guidance led to multiple efforts at providing a framework for concussion management via consensus statements or systematic reviews of the literature. Most recently, efforts by the international Concussion in Sport Group (CISG), the American Medical Society for Sports Medicine (AMSSM), and the AAN, among others, have led to such documents, all designed to help the clinician navigate best practices while admittedly being based on relatively sparse published data. ${ }^{10-12}$ (Refer to Appendix A for a summary of the AAN's evidence-based guideline for clinicians.)

It should be noted that, while the term concussion may refer to a very specific injury construct or a welldescribed clinical syndrome, the issue of concussion encompasses much more, both in the public eye and in the practice of medicine. It has become increasingly clear that the clinician cannot provide a high standard of clinical care to the concussed patient by considering only the ramifications of the acute injury. One must also consider the risk factors for chronic conditions such as postconcussion syndrome and the potential for fixed neurocognitive impairment or progressive neurodegenerative disease, a clinical task addressed only via a comprehensive neurologic evaluation. As a direct response to the complexity of these clinical decisions and increasing public awareness, neurologists are finding themselves more directly involved in sport concussion care than ever before, and in every conceivable setting, from the sidelines of sporting events to the laboratory, athletic training rooms, and outpatient clinics.

The presence of other diagnostic entities such as postconcussion syndrome and CTE in the clinical schema of the concussed athlete highlights the important role neurologists are well suited to play in sports concussion management. Traditionally,
BrainSPORT program from Steve Tisch.

Unlabeled Use of

Product/Investigational

Use Disclosure:

Drs Kutcher and Giza report no disclosure.

(C) 2014, American Academy of Neurology. 


\section{KEY POINTS}

- Concussion is a complex pathophysiologic response to biomechanical forces imparted to the brain, producing a transient constellation of neurologic symptoms that reflect a functional rather than a structural injury.

- Concussion may be present at the same time as more structurally based intracranial injuries induced from a biomechanical force. neurologists in training are extensively exposed to pain disorders, such as migraine headache, and dementing illnesses, such as Alzheimer disease. Understanding these classic neurologic diagnoses is directly relevant to the evaluation of the concussed athlete and provides the neurologist with a unique perspective on the overall neurologic health of these patients. Despite the field's long history with this injury, however, it was not until recently that concussion itself has become a classic neurologic diagnosis.

\section{DEFINITION OF CONCUSSION Pathologic Effect Versus Clinical Syndrome}

Concussion has been defined as a complex pathophysiologic response to biomechanical forces imparted to the brain. ${ }^{10,13}$ It has also been described as a transient constellation of neurologic symptoms that reflect a functional rather than a structural injury. ${ }^{4}$ In most definitions, whether referring to concussion as an injury process or a clinical syndrome, further care is taken to delineate concussion from more severe traumatic brain injuries (TBIs), classically those that involve a Glasgow Coma Scale score of 12 or less. For this reason, the term mild TBI has largely been used interchangeably with concussion. Another frequently stated stipulation for the diagnosis of concussion is the lack of acute abnormalities seen on routine neuroimaging (CT or standard MRI). This point is often mistakenly applied to conclude that the presence of an acute abnormality on routine neuroimaging, such as hemorrhage, precludes the diagnosis of concussion. In actuality, concussion may be a concurrent result of the imposed biomechanical force, existing alongside more structurally obvious pathologies.

The pathophysiology of concussion continues to be elucidated. Currently, the clinical syndrome of concussion is presumed to be the result of biomechanical forces inducing a complex metabolic cascade involving impaired neurotransmitter function, excitotoxicity, and grossly abnormal concentrations of intracellular and extracellular ions. ${ }^{14}$ Most of these effects have been shown to last several hours to multiple days in animal models. Axonal stretching effects have also been implicated, but the exact role of this mechanism in concussion is unclear. Finally, either as the result of the above physiologic perturbations or potentially as an independent but related pathophysiologic process, significantly decreased cerebral blood flow, approximating $50 \%$ of normal, is also seen for several days following experimentally induced concussion in animals. ${ }^{15}$

It should be clearly noted that the brain can experience the necessary biomechanical force to induce this cascade without coming in contact with the inside of the skull and without the head itself coming in contact with the insulting object. The only biomechanical prerequisite for injury is the presence of a large enough acceleration/deceleration force. Forces significant enough to induce the metabolic cascade described above can result from a blow to the body that subsequently causes a whiplash movement of the head and neck.

Armed with the above pathophysiologic construct of concussion, the clinician should take great care in applying a critical thought process to each clinical scenario. The lack of a readily available objective test demonstrating the presence of the physiologic injury state described above means that concussion remains a clinical diagnosis. From this distinction, the term subconcussive injury has been used to describe a subset of mild TBI that does not produce a clinical syndrome. ${ }^{12}$ This distinction does have a significant role to play clinically. A 
patient who has experienced a blow sufficient to induce the metabolic cascade of concussion and then goes on to have a notable clinical syndrome should be easily diagnosed with concussion after the appropriate neurologic evaluation. If, however, such an evaluation does not identify a clinical effect, the clinician cannot conclude that the patient is not injured, as it may be the case that the underlying pathology is not sufficient to produce a clinical effect. Thus, when considering concussion as a clinical syndrome, the clinician should realize that the production of symptoms, and therefore the ability to diagnose the presence of the injury, is a process dependent not only on the pathology itself, but also on that particular brain's ability to produce symptoms when injured. At times, it may be more appropriate to describe concussion as the underlying injury itself, and at other times, it may be best to frame concussion as the clinical syndrome produced by the underlying pathophysiology. In most cases, however, it is best to keep both in mind equally.

\section{Concussion as a Network Injury}

When defined, neurologic injuries or diseases are often described initially by the distribution of pathologic effect, noting whether the pathology is focal, multifocal, or diffuse. The pathologic process of concussion can best be described as a diffuse process involving the brain. The clinical presentations of concussion are heterogeneous and, not surprisingly, difficult to localize to one specific anatomic location. The diffuse nature of the injury, rather, produces clinical syndromes that often involve the disruption of functional brain networks, such as those responsible for memory, balance, and vestibular control. In this way, concussion can be considered to be a diffuse pathologic process that produces brain network dysfunction.

\section{Clinical Definitions: Possible, Probable, and Definite Concussion}

As noted above, no reliable objective test exists for the diagnosis of concussion. Therefore, clinicians must rely on their skill and experience in making a clinical neurologic diagnosis. ${ }^{10-12}$ In the end, however, the diagnosis of concussion may not always be clear, regardless of the quality of the evaluation and the skill of the examiner. Such cases are likely growing in number as public awareness of concussion continues to increase, especially when noting that the common symptoms of concussion are not specific as to cause. In these instances, the clinician must make an additional determination beyond whether concussion is present or not. To deliver the best possible care, one must also decide whether or not that patient should be managed as if he or she has a concussion. To aid in that decision, it can be extremely helpful to further classify concussion diagnoses by degree of certainty. Table 2-1 describes the characteristics of three categories proposed by the authors for determining a degree of certainty in concussion diagnosis.

Possible concussion. In some cases, patients being evaluated for concussion have multiple potential symptomgenerating factors, and, in the end, concussion is not felt to be the most likely cause of their clinical syndrome. In these cases, a clearly identifiable alternative explanation exists for the clinical presentation. Additionally, a biomechanical mechanism of concussion may not be clearly established. These patients can be considered to have a possible concussion. As an example, it is very common for athletes to develop symptoms of headache and fatigue during vigorous activity, due only to the level of exertion itself, with

\section{KEY POINTS}

- Diagnosing concussion is dependent not only on the pathology itself, but also on that particular brain's ability to produce symptoms when injured.

- Concussion is a diffuse pathologic process that produces brain network dysfunction. 


\section{TABLE 2-1 Concussion Diagnosis by Degree of Certainty}

\begin{tabular}{ll} 
Classification & Definition \\
\hline Possible & $\begin{array}{l}\text { Concussion is not the most likely cause of } \\
\text { the clinical presentation. Other possible } \\
\text { explanations are identified, such as } \\
\text { migraine, dehydration, or viral illness. } \\
\text { The presumed traumatic insult was not } \\
\text { witnessed or is difficult to describe. }\end{array}$ \\
Probable & $\begin{array}{l}\text { Concussion is the most likely cause of the } \\
\text { clinical presentation. While other possible } \\
\text { explanations exist, they are deemed less } \\
\text { likely. The traumatic insult was clearly } \\
\text { defined by witnesses or is identifiable } \\
\text { on video. }\end{array}$
\end{tabular}

Definite
Concussion is the only explanation for the clinical presentation.

\section{Management}

Situational (clinician may decide to treat as concussed or not, depending on the specific clinical situation)

As concussed

As concussed or without a contribution from a preexisting or coexisting condition such as migraine headache or hyperthermia. If this scenario occurs in the setting of a contact practice, it may then wrongly be assumed that a biomechanical insult was responsible for the symptoms, whether or not a significant impact exposure was identified. The clinician may treat these patients as if they are concussed, or not, depending on the level of comfort the clinician has in making an alternative diagnosis. Deciding to treat a patient with possible concussion as if he or she is not concussed is a difficult decision and should be made in close cooperation with the patient's athletic medicine staff. Close observation and frequent daily evaluation of these patients may be required.

Probable concussion. In other cases, a concussive mechanism is suspected and felt to be the most likely explanation for the clinical presentation, despite the presence of other causative diagnoses. These cases are best described as probable concussion. Often, concussion and nonconcussion pathologies coexist and combine to form the overall clinical presentation. Common examples of exacerbating or coexisting pathologies include migraine headache, sleep disturbance, cervical spine pathology, anxiety or other mood disorders, and attention deficit disorders (ADHD). In these cases, patients should be treated as if they are concussed, while also being carefully evaluated for the appropriate treatment of any other present condition.

Definite concussion. Finally, patients who experience a clear loss of consciousness from a witnessed biomechanical trauma can be considered to have a definite concussion. In the absence of a clear loss of consciousness, the observation of posturing in a patient, if made by a clinician trained in the identification of this physical sign, may also be used to reach the level of clinical certainty needed for a definite concussion determination. In most cases of definite concussion, however, neither loss of consciousness nor posturing is observed. Rather, a clear clinical syndrome of concussion exists, in the absence of any other reasonable explanation for symptoms. 


\section{DIAGNOSIS}

\section{Common Signs and Symptoms of Concussion}

The common signs and symptoms of concussion are presented in Table 2-2. Although commonly linked with concussion in public perception, loss of consciousness is not a requirement for concussion diagnosis. In fact, significantly less than $10 \%$ of diagnosed concussions involve a loss of consciousness. ${ }^{10-12}$

\section{TABLE 2-2 Common Signs and Symptoms of Concussion}

\section{- Cognitive/Mental Status \\ Inattention}

Slowed thinking

Amnesia

Confusion

Disorientation

Vacant stare

Loss of consciousness

\section{- Physical}

Headache

Nausea/vomiting

Photophobia

Phonophobia

Dizziness

Slurred speech

Blurred vision

Incoordination

\section{- Affective}

Emotional lability

Depression

Anxiety

Mania

\section{- Sleep}

Increased latency

Frequent waking

Increased sleep time

Decreased sleep time
Some degree of anterograde or retrograde amnesia is far more common in concussion than loss of consciousness, occurring in an estimated 30\% to 50\% of concussed patients, while headache is the most common symptom reported overall. ${ }^{16}$ Concussed patients often present with a clinical syndrome similar to those seen in other neurologic patients. For example, concussion patients often appear and behave similarly to those having an acute migraine headache. A significant degree of shared phenotype also exists with patients carrying a diagnosis of toxic-metabolic encephalopathy. Physical symptoms of imbalance and dizziness will remind the neurologist of patients with central or peripheral causes of vertigo. The transient memory dysfunction seen in concussion is similar to that seen in transient global amnesia. Finally, concussed individuals will often present with a mental status very similar to that seen in patients with a wide variety of mood disorders. In concussion, the unifying theme to the natural history of any sign or symptom is the commonly seen time course of lasting several days to 2 weeks with gradual, yet complete, symptom resolution in the majority (approximately 85\%) of cases. ${ }^{10-12}$

\section{Concussion Diagnosis Over Time}

Concussion is an injury resulting from an acute biomechanical force or series of forces that impart their effects on the brain on the time scale of milliseconds. Given the evolving metabolic nature of the subsequent pathology and the need for a clinical syndrome to develop prior to identification of injury, however, it is often impossible to diagnose concussion immediately following the biomechanical insult. It has been noted that the signs and symptoms of concussion may take several hours to develop or may not be noted by the patient until further cognitive or physical exertion

\section{KEY POINTS}

- Loss of consciousness is not a requirement for concussion diagnosis. In fact, significantly less than $10 \%$ of diagnosed concussions involve a loss of consciousness.

Some degree of anterograde or retrograde amnesia is far more common in concussion than loss of consciousness, occurring in an estimated $30 \%$ to $50 \%$ of concussed patients, while headache is the most common symptom reported overall. 


\section{CONTINUUM Sports Concussion}

\section{KEY POINT}

The signs and symptoms of concussion may take several hours to develop or may not be noted by the patient until further cognitive or physical exertion is experienced. is experienced (Case 2-1). ${ }^{10-12}$ Therefore, clinicians must remain vigilant and adaptive in their diagnostic evaluation, allowing for the possibility of evolving diagnostic certainty.

\section{Differential Diagnosis}

Many common neurologic processes, both pathologic and within the range of normal human function, may produce symptoms and presentations similar to concussion. During each step in the concussion management process, care should be given to consider all possible symptom generators. Common comorbid conditions include migraine, sleep disorders, anxiety, cervical spine disease, and attention disorders. Often, patients experience symptoms both from concussion and one of these processes concurrently, adding to the complexity of clinical management.

\section{Diagnosis and Management}

As noted above, the diagnosis of concussion is made on a clinical basis. Subsequent concussion management, therefore, is best dictated by frequent serial evaluations. An overall approach to concussion diagnosis and management is shown in Figure 2-1 and Figure 2-2. The approach presented begins with a preinjury neurologic assessment, typically in the preseason. When a concussion is then suspected, an initial concussion assessment is performed. Once diagnosed, concussions are best managed by using serial evaluations to determine the absence of any concussion signs or symptoms at rest. Then, the patient proceeds through a graduated exertion process (further defined below) that ultimately leads to the return-to-play

\section{Case 2-1}

A 17-year-old ice hockey player reported headache and light sensitivity to his parents following a game. He had no cognitive complaints other than feeling "foggy." He gave clear, detailed, and correct information regarding the events of the game. He recalled no one extraordinary hit but did describe the game as being very physical; he was a defenseman who played with a physical style. His headache was further described as dull, constant, and occipital in location, with some component of upper cervical discomfort. He mentioned that after some games, whether he had experienced significant contact or not, he had similar but less severe versions of his current headache. His symptoms continued for the rest of the day and evening. His appetite was unaffected. His sleep was typical for him that evening. The next morning, his headache continued, but was milder, and he felt that it was more clearly located at the top of his neck. Concerned for concussion, his parents took him to his primary care physician the next day. Neurologic examination was performed and found to be normal, other than diffusely decreased cervical range of motion and mild convergence insufficiency.

Comment. Headache and neck discomfort are common complaints in athletes, especially those participating in a collision sport such as ice hockey. Athletes will typically present with occipital nerve pain following significant physical contact. Additionally, while vague mental status symptoms such as feeling "foggy" should make one consider concussion, other potential explanations may exist. Both symptoms are nonspecific and may be related to concussion, a primary headache disorder, physiologic state, or musculoskeletal insult. No objective diagnostic test can confirm the presence of concussion. Physical examination findings, such as convergence insufficiency, may be clues but are not diagnostic. The diagnosis of concussion is best made based on careful formulation of information collected via a comprehensive neurologic evaluation. Even then, it is often not obvious that a concussion has occurred. In these cases, it is best to describe the possibility of all identified symptom mediators while taking care to design a management approach that includes concussion as a possibility. In this case, it would be appropriate to diagnose a possible concussion. Other potential causes, such as occipital neuralgia or muscular strain, should also be considered. The diagnosis of concussion may be clarified over time, however, as the symptom complex evolves. 


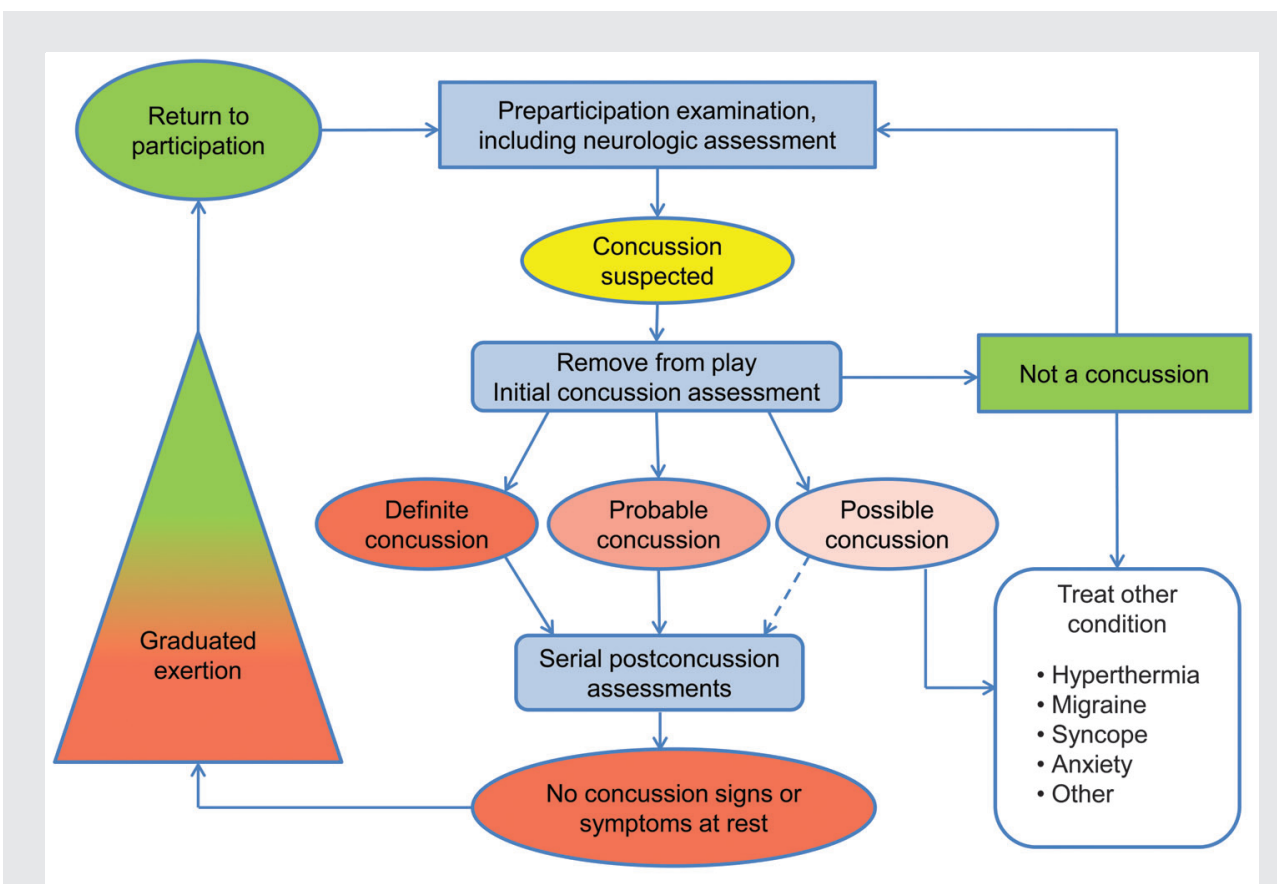

FIGURE 2-1 Approach to concussion diagnosis and management.

decision. Whether evaluating a player in the preseason or postinjury, the clinical approach to evaluating a patient should be similar.

Preinjury clinical neurologic assessment. Concussion management often begins well before the patient is injured. Preparticipation examinations are an opportunity not only to determine an athlete's fitness to play, but also to collect information on past medical history and family history that may prove useful in future injury management. Preparticipation examinations are extremely common in sports medicine and are mandated at many levels. Whenever possible, the preparticipation examination should include a comprehensive neurologic assessment that focuses not only on concussion

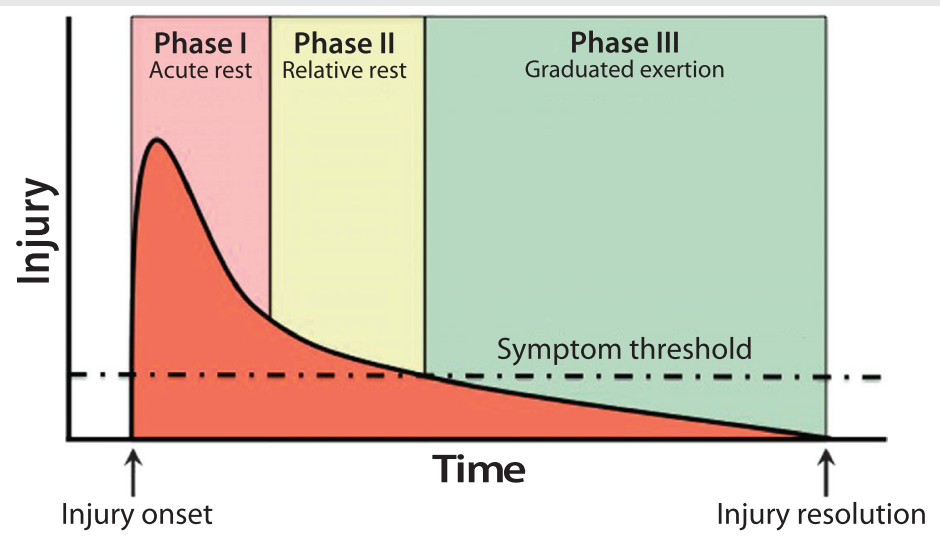

FIGURE 2-2 Three phases of concussion management. 


\section{KEY POINT}

- The appropriate use of preinjury clinical tools is as a source of data to be included as part of a comprehensive neurologic evaluation. history, but also on the athlete's personal and family overall neurologic history, including migraine, attention and learning disorders, sleep disturbances, mood disorders, and neurodegenerative diseases. A comprehensive neurologic examination should be performed with the intent of performing the same examination in future injury scenarios.

It is becoming increasingly common to employ preinjury or "baseline" clinical testing for athletes as part of a sport concussion management program. Simply put, the idea is to obtain an objective measurement of brain function in the noninjured state for use as a comparison tool to aid in concussion management. The use of the term baseline is common but may be somewhat misleading. Given the complexities of brain function and the tremendous number of variables that can affect it, the results of any preinjury clinical test should only be considered a snapshot of that individual's performance, rather than a true representation of the patient's innate best ability. Common factors that may influence the results of preinjury testing include the testing environment, subject motivation, sleep deficit, pain, and anxiety. It should also be noted that some patients may have employed less than complete effort in the preinjury setting in order to obtain a low score, thus making it easier to return to their preinjury level of performance while in the injured state.

It is also common that these tests are misused clinically, being treated as diagnostic tools or return-to-play determinants. Their appropriate use, rather, is as a source of clinical data to be included as part of a comprehensive neurologic evaluation. Commonly used types of preinjury testing can be divided into those that evaluate a purely physical task, those that evaluate a purely cognitive task, and those that include some aspect of both. Commonly used physical evaluation tools include the clinical reaction time "stick drop" test $\left(\mathrm{RT}_{\text {clin }}\right)$, balance tests such as the Balance Error Scoring System (BESS), and the King-Devick Test of visual tracking. ${ }^{17-19}$ Common preinjury cognitive tests include computerized tools such as the Axon Sports Computerized Cognitive Assessment Tool (CCAT) and the Immediate Post-Concussion Assessment and Cognitive Testing (ImPACT) assessment tool, and paper-and-pencil tools such as the Standardized Assessment of Concussion (SAC). ${ }^{20-21}$

Many sports organizations at every level of play now mandate the use of preinjury testing. Therefore, clinicians frequently caring for concussed patients should familiarize themselves with the commonly used tests and note that each test is unique onto itself, possessing specific statistical properties and open to varying degrees of effects from the potential confounding variables mentioned above. Overall, no one preinjury evaluation tool has been shown to be superior over another.

Evaluation on the field of play. Increasingly, neurologists are being asked to provide acute concussion evaluations during sports competitions and practices. Even if the practicing neurologist does not have a sideline responsibility, knowledge of the sideline process and environment can be helpful in providing comprehensive concussion care. In either case, it should be recognized that the approach to sideline evaluation and management is unique in many ways.

Prior to any sideline assignment, the emergency action plan for that location and the teams involved should be reviewed. In most cases, the neurologist is not the sole medical provider on-site. Establishing roles and responsibilities, determining the location of all emergency equipment, and establishing a clear line of communication are essential. Often, at least one athletic trainer is present. Athletic trainers (often called athletic therapists in countries other than 
the United States) are medical care providers specifically trained in the triage and management of acute sports injuries of all types. Athletic trainers are an invaluable resource for the sideline physician. They are often the point of contact for coaching staff and parents. If they are employed by a particular team or institution, their preinjury knowledge of athletes is particularly important, as they can provide insight into the more subtle mental status findings present in some concussed patients. Their knowledge of patients' past medical history and their relationship with parents or guardians can also be extremely beneficial.

When attending a sporting event or practice, it is helpful to be aware of the locations that provide the best sight lines for observation and access to potentially injured athletes. While it may be impossible to watch every athlete in a continuous fashion during a sporting event, experienced sideline physicians should anticipate potentially injurious contact and place themselves accordingly. If a concussion is suspected, the first medical responsibility is for the patient's safety. If play is ongoing and represents a significant risk to the injured player or the medical staff, the officials or coaches overseeing the event should be notified immediately. Medical assessment can then begin with assessment of the "ABCs" (airway, breathing, circulation). If any of these are compromised, emergency medical services should be activated immediately. Other factors that may warrant activation of emergency medical services include a prolonged loss of consciousness, seizure, suspicion or evidence of cervical spine disease, or a rapidly deteriorating level of consciousness. Of course, the care provider should always err on the side of safety when contemplating emergency actions.

The next step is to relocate the patient to a safe and calm environment in order to perform a concussion evaluation. In these settings, it is always preferable to have a well-structured approach to the evaluation. The Sport Concussion Assessment Tool 3 (SCAT3) is a product of the 4th International Conference on Concussion in Sport and is intended to provide an organized framework for performing a neurologic sports concussion assessment. The SCAT3 is not intended to be used in its entirety on the sideline. ${ }^{10}$ Rather, the initial sideline assessment portion of the SCAT3 was developed for this purpose (Table 2-3). This initial assessment acts as a concussion screening evaluation. If none of these potential signs are present but suspicion for concussion continues, the clinician should then expand the evaluation to include a more thorough and comprehensive evaluation. Also, as previously noted, the clinical syndrome of concussion may take several hours to develop, necessitating serial evaluation. In this way, the process of diagnosing concussion is often one that occurs over several hours.

If a concussion is diagnosed, the patient should not return to sport participation for the remainder of the day, mainly to limit the degree of metabolic injury and to provide patient comfort. ${ }^{10-12}$ This is also a reflection of the legal precedents set forth by the state level youth concussion laws now enacted in all 50 US states. Details of state concussion laws can be found in the AAN Sports Concussion Toolkit. ${ }^{22}$ (For a complete list of resources included in the Sports Concussion Toolkit, see Appendix B.) While each of these laws has its own language and legal implications, one consistent theme is that no youth athlete diagnosed with concussion should return to play on the day of diagnosis. These laws were designed, in large part, to limit cases of second impact syndrome, despite the extremely low incidence of this diagnosis and substantial questions as to the appropriate criteria and framework for describing the injury.

\section{KEY POINTS}

- When considering activating emergency medical services, always err on the side of safety.

- The ability to diagnose concussion may be delayed for several hours, when signs or symptoms eventually develop. 


\section{TABLE 2-3 Sideline Assessment Portion of the Sport Concussion Assessment Tool 3}

- Indications for Emergency Management

Glasgow Coma Scale score less than 15

Deteriorating mental status

Potential spinal injury

Progressive, worsening symptoms or new neurologic signs

\section{- Potential Signs of Concussion}

Any loss of consciousness (If so, how long?)

Balance or motor incoordination

Disorientation or confusion

Loss of memory (If so, how long? Before or after the injury?)

Blank or vacant look

Visible facial injury in combination with any of the above

After being removed from participation, the patient should be made comfortable in a distraction-free environment if possible. Often, nausea, photophobia, and phonophobia are present, and efforts should be made to minimize environmental exacerbation of these symptoms. The concussed athlete should remain under direct observation, however, to provide the opportunity for vigilance regarding worsening mental status (Case 2-2). Observation should

\section{Case 2-2}

A 14-year-old female soccer player made contact with an opponent's head while attempting to head the ball during a game. She was knocked off balance from the impact and landed on the ground, striking her head a second time. She was witnessed to be unresponsive with her eyes closed for approximately 10 seconds. She then regained awareness but was clearly disoriented. She was evaluated by her team's athletic trainer, and no emergent condition was identified. She reported headache and nausea and did not play the remainder of the game. After 10 minutes, she was oriented but had incomplete memory for the events surrounding the injury and slowed verbal responses. Over the next 2 hours, she was observed on the sidelines, and serial mental status examinations were performed by the athletic trainer. Other than an increasing sensitivity to light and sound, no changes were noted. Her parents arrived after the game and immediately took her to the emergency department of a local hospital, where she was observed further. A CT scan of her head was performed and found to be normal.

Comment. Loss of consciousness after a noted head impact is a clear sign of brain dysfunction. Concussion is easy to diagnose in these cases, but initial medical evaluation should be directed at ruling out a more serious intracranial injury such as hemorrhage. If concussed athletes are medically stable, they should be observed for clinical worsening. Often, it is preferable for patient comfort to relocate them to a quiet distraction-free environment. This should not be done, however, at the expense of continued observation. Not all concussive events require emergent evaluation, but it can be the best course of action if the available resources do not allow for serial medical evaluation. Head CT does not provide information about the presence or absence of concussion and should only be used if, based on the clinical presentation, an evaluation for hemorrhage or fracture is warranted. 
continue until the clinician feels comfortable that any substantial risk of a concurrent intracranial hemorrhage or other emergent pathology has passed. Typically, this is at least a 4-hour period. If it is not possible to provide adequate medical observation, the patient should be transferred to the closest urgent care or emergency department. If an emergency department evaluation is undertaken, the lack of clinical utility of routine neuroimaging in diagnosing concussion should be noted. These modalities should be used, however, when clinical judgment regarding the potential for additional urgent intracranial pathology dictates.

In the acute setting, it is best to avoid anti-inflammatory pain medications in order to minimize bleeding risk as well as any medications that may be reasonably expected to produce an effect on the patient's mental status. It is reasonable, however, to consider acetaminophen for the initial control of headache within the first few hours. Antiemetic medications may be considered as well, although, again, care should be taken to avoid medications that might alter the patient's mental status. Arrangements should be made as soon as possible with family, friends, or team personnel to ensure that the patient will not be alone for the next several hours. For the remainder of the day, the patient should be told to rest, both physically and cognitively, as symptoms determine. If nausea is not present, appetite may still be suppressed. Patients should be told to drink water and attempt to ingest their typical caloric intake. Sleep is quite commonly affected in concussion, especially during the first evening. Increased sleep latency, frequent waking, and inefficient sleep are all common. Patients should be counseled about this likelihood, but the use of sleep aid medications should be limited initially.
Finally, a follow-up plan should be determined, with emphasis on a comprehensive neurologic follow-up, within 24 hours if possible, but as soon as practical otherwise.

Evaluation in the clinic. Until recently, neurologic referral of a concussed athlete occurred in a clear minority of cases, typically those with symptoms lasting longer than 4 weeks or cases with a complicating factor such as posttraumatic seizure, prolonged loss of consciousness, or structural intracranial pathology. It is becoming increasingly common, however, for neurologists to be asked to evaluate an athlete in clinic shortly after a concussion has been diagnosed and then to be directly involved in their early management. Early involvement in these cases provides the opportunity for a comprehensive neurologic approach to the return-to-play decision, management of common neurologic comorbidities, and appropriate consideration of potential long-term consequences. Many of the core principles of sports concussion diagnosis and management are similar to those seen in other common neurologic diagnoses and, thus, should be quite familiar to the practicing neurologist.

The initial approach should be to verify the diagnosis of concussion. With heightened awareness and concern regarding concussion comes a natural, and arguably appropriate, increase in false-positive diagnoses. It can be considered a neurologic axiom that not every symptom complex experienced by a patient following a contact play in sports is caused by concussion. Thus, the differential diagnosis in these cases should include other common mechanisms, such as migraine, cervical muscle strain, and anxiety. At the same time, one must always consider the possible existence of an additional intracranial process such as hemorrhage. In a very general sense, the timing and severity

\section{KEY POINTS}

- Pain medications should be avoided immediately following a concussive event.

Not every symptom complex experienced by a patient following a contact play in sports is caused by concussion. 
of symptoms is key to making these determinations. Concussion symptoms tend to be maximal minutes to hours after the impact in question and then resolve slowly and progressively over the subsequent 7 to 10 days. ${ }^{10}$

While migraine headaches often have variable timing and presentations, they are typically shorter in duration, with more severe symptoms and a more obvious and sudden resolution when compared with the typical clinical syndrome of concussion. If the patient has a history of migraine, the current presentation should be compared closely with his or her typical migraine presentation.

It is quite common for sleep to be significantly impaired or disrupted in the first few days after a concussive event. Sleep difficulties may then be further exacerbated by anxiety and the dramatic decrease in activity typically experienced in the early stages of concussion. In cases of significant sleep disruption, the clinician should carefully consider how much of the clinical presentation may be from sleep effects alone.

It is not uncommon that patients with concussion describe worsening symptoms for several days following the initial injury, but typically these exacerbations can be correlated with situational explanations, such as significant cognitive or physical exertion or exposure to noxious environments, and they are also typically short-lived. In these cases, taking a step back and considering the overall temporal trend, the clinician can often discern a pattern of improvement that is punctuated with brief, transient periods of worsening. In contrast, if after 24 hours the concussed patient is noted to have a progressively worsening level of consciousness, dramatically worsening headache, or any new neurologic deficit, an intracranial process should be ruled out and a head CT should be performed emergently.
After the history is taken, the neurologic examination can then be performed, first focusing on those specific neurologic systems described in the literature as frequently affected in concussed patients. These include an evaluation of cognitive function, eye movements, balance, gait, and coordination. ${ }^{13,14}$ Once other symptomatic mechanisms have been addressed via a thorough history and examination, the clinician can then decide if concussion is an appropriate diagnosis. As noted above and described in Table 2-1, it may also prove useful to further describe the injury as possible, probable, or definite, providing further clarity into the complexity of the clinical scenario and helping to set appropriate expectations.

The next step is to consider the degree to which cognitive and physical activities should be minimized or avoided. A clear consensus exists in clinical practice recommendations that some degree of rest from both cognitive and physical activities is recommended for a period of time. ${ }^{10-12}$ What decrease in activity level is necessary for a definition of rest and for how long the patient should be at this level is not well understood or described, however. More recently, recommendations have begun to reflect the concept of "relative rest," a degree of activity that is reached by careful evaluation of symptom exacerbation brought on by any particular activity. ${ }^{23}$ This idea can be simply stated as "rest as needed" for symptom control. It should then also be noted that, in some patients, dramatically decreasing their level of cognitive, physical, and, in many cases, social activity may produce detrimental effects as well, leading to sleep difficulties, exacerbations of mood disorders, and even physical symptoms such as headache. Thus, 
while the prescription of rest in the period immediately following concussion onset is likely to be of some benefit, it may significantly cloud the clinical picture by producing symptoms that mimic concussion. This competing concept can be similarly simply stated as "rest as tolerated." Therefore, rest should be considered an intervention best used "as needed and as tolerated." In the end, the neurologist should carefully consider the overall clinical picture and suggest adjustments to the patient's schedule that reflect acknowledgment of these two competing concepts. Often, academic accommodations such as limiting classroom hours in a day and providing extended time for assignments and examinations are necessary to help the patient tolerate school activities while also having the student athlete avoid falling too far behind academically.

Once a plan for the appropriate level of activity has been determined, the ongoing symptoms of the clinical concussion should then be addressed. Symptoms such as headache, nausea, and sleep disturbance can be addressed with medications as they would in nonconcussed patients. When deciding on when and how aggressively to begin a return-to-play process, the degree to which any current medication use is masking the symptoms of concussion should be carefully evaluated.

To further organize the clinical approach, each of the individual aspects of concussion management described above can be framed into three distinct management phases, presented in Figure 2-2. This theoretical framework is based on the concept of the symptom threshold below which a patient is still injured but not producing a detectable clinical syndrome. It is further based on recent animal data that demonstrate a win- dow of time following concussive injury during which initiating physical activity is found to provide the best recovery curve. ${ }^{24}$ The overall goal of this approach is to accurately identify when the metabolic injury itself is over so that the patient can be returned to full sports participation safely. Each phase is defined by the current clinical experience of the patient. Currently, no published data exist that speak to the clinical utility of this approach, but the authors have found it useful in clinical practice. The time spent in each phase is highly variable but approximations are provided below. These time frames are only estimates and should not be used in isolation to determine the appropriate phase of management. Time windows may be longer in younger athletes or those with complicating comorbid diagnoses. (Refer to the article "Pediatric Issues in Sports Concussion" by Christopher C. Giza, MD, in this issue of CONTINUUM .)

Phase I ( 0 to 5 days). The patient experiences acute symptoms that, because of their severity, significantly limit both cognitive and physical activities. During this "acute rest" phase, the patient is typically unable to tolerate routine activities of daily living, and the physician can comfortably recommend significant rest as a cornerstone of treatment. This is also the best time to set expectations for recovery. Symptomatic use of medications is reasonable in this phase.

Phase II (2 to 10 days). The patient is still symptomatic, but not to a degree that consistently limits his or her participation in routine activities. At this time, the patient should begin returning to the preinjury academic and social schedule, but should only engage in activities to a degree that does not significantly worsen symptoms. During this phase, the use of medications for

\section{KEY POINT}

- Acute rest may produce symptoms similar to those caused by concussion. 


\section{KEY POINT}

- The return-to-play decision starts with determining that the underlying metabolic injury of concussion is no longer present. the control of concussion symptoms should begin to be tapered and then limited, as entrance to the next phase of management requires patients have no notable clinical syndrome of concussion while off medications.

Phase III (7 to 14 days). The patient no longer experiences the signs or symptoms of concussion after returning to routine levels of daily activity. At this stage, the patient should not be using medications for the treatment of concussion-related symptoms. The main point of this stage is initiating the active process of return to play, which requires exposure to a graduated paradigm of increasing physical exertion. This is done to move the symptom threshold down, so that a brain still experiencing the metabolic injury of concussion will be more likely to produce a clinical syndrome. If the patient does not experience a recrudescence of concussion symptoms during this process, it can be interpreted as clinically suggesting that the metabolic injury itself is over.

\section{RETURN TO PLAY}

The return-to-play decision is perhaps the one aspect of sports concussion care that sets it apart from non-sportrelated injury. This is because the physician is determining when it is appropriate for the patient to return to the same activity that caused the injury and has an intrinsic risk of subsequent injury or complications. Therefore, at the heart of making any return-to-play decision is the process of determining as confidently as possible that the underlying metabolic injury of concussion is no longer present. ${ }^{10-12}$ Given the absence of a direct method for measuring the pathophysiology of the injury, this is best accomplished by having the patient who reports no signs or symptoms of concussion with routine activity begin the process of graduated exertion (Table 2-4).

Prior to beginning this process, it is extremely important to understand the degree of oversight or direct observation the patient will be under. In some cases, an athletic trainer or other sports medicine staff person is able to supervise these activities directly. Alternatively, as neurology clinics evolve to accommodate this population, the patient may be able to return to the neurologist's clinic for direct observation during physical exertion. In contrast, many patients will be doing this on their own or under the guidance of a parent or coach. Each situation is different, and clinical judgment must

\section{TABLE 2-4 Steps of the Graduated Exertion Process ${ }^{a, b}$}

Step ${ }^{c} \quad$ Notes

1. Light aerobic exercise

2. Sport-specific aerobic exercise

3. Noncontact training drills

4. Contact training drills

5. Return to play

${ }^{a}$ Modified with permission from McCrory P, et al, Br J Sports Med..$^{10}$ (c) 2013, BMJ Publishing Group Ltd and the British Association of Sport and Exercise Medicine. bjsm.bmj.com/content/47/5/250.full.

${ }^{b}$ To be initiated when the patient is no longer experiencing concussion-related signs or symptoms with routine activities of daily living.

' Proceed to next step only after enough time has passed to clinically determine the absence of sign or symptom recrudescence. 
be used to determine the appropriate rate of graduating from one step to the next. If any preinjury or postinjury clinical testing was performed, the patient's postinjury performance should either be back to his or her preinjury level or be within population-based norms for each test if a preinjury result is not available. If the patient shows a noted decrement in performance, or the postinjury testing falls below the expected standard deviation, the patient should be considered still injured, unless a reasonable alternative explanation exists, such as medication use, concurrent illness, or acute physiologic state.

Each stage of the process is intended to last as long as the clinician feels necessary to assess for absence of symptom recrudescence in each individual patient. Typically, this results in the patient being at each stage for 24 to 48 hours, although no specific data speak to this timing. If signs or symptoms do reappear and are felt to be directly related to the concussion injury, the patient should rest for the remainder of that day and return to the previous level once the signs and symptoms have resolved once more. Again, every situation is unique, and clinical judgment should ultimately decide the rate of progression. By the end of the process, the physician should feel confident that the patient has been exposed to the physical and cognitive rigors of competitive sport activity, short of being exposed to contact situations, without producing any signs or symptoms of a concussive injury. Only then can it be assumed to be safe to return to full participation.

\section{REASONS FOR RETIREMENT FROM CONTACT SPORTS}

Unfortunately, sparse data exist in the published literature to help the neurologist make this difficult recom- mendation. To the best of their clinical ability, neurologists should assess the risk of prolonged or permanent neurologic effects from continued participation in sports while also carefully considering the positive aspects to continuing. It should be stressed that no clear number of diagnosed concussions has consistently been shown to have any effect on any particular long-term outcome. To that end, consensus is growing that the number of diagnosed concussions is less important than the individual's lifetime exposure to impact forces when describing longterm brain health risks. ${ }^{9}$ In general, three scenarios warrant a closer look at recommending retirement from contact sports.

The first is a clear lowering of the threshold for injury. In some cases, one can determine to a reasonable degree of certainty that, over time, less force is required for a patient to acquire a concussion. Caution should be used, however, because of the subjective nature of concussion presentations and the fact that common concussion symptoms are nonspecific as to cause. For these reasons, it may also be the case that a previous diagnosis of concussion makes it more likely that concussion becomes the default diagnosis for subsequent clinical syndromes, whether the current clinical presentation is due to concussion or not. Nonetheless, if a pattern of lowering threshold for injury is suspected, retirement from contact sports is at least a reasonable recommendation.

The second scenario is found in patients whose clinical experience with concussion consistently produces a degree of morbidity that outweighs the benefits of playing the sport in question. In other words, although most concussions will last 7 to 10 days on average, some individuals, when injured,

\section{KEY POINTS}

- Clinical judgment should be used to determine the appropriate rate of completing the graduated exertion process.

Each stage of the graduated exertion process is intended to last as long as the clinician feels necessary to assess for absence of symptom recrudescence in each individual patient.

- If a pattern of lowering threshold for injury is suspected, retirement from contact sports should be strongly considered. 


\section{KEY POINT}

- Objective signs of declining brain function thought to be secondary to impact forces warrant retirement from contact sports. consistently produce a clinical syndrome that is more severe than average and may last for several weeks. In addition, there may be a predilection for developing a postconcussion syndrome that warrants further consideration. The relative contraindications for playing a sport should be weighed carefully against the personal benefits the patient experiences from playing that sport.

The third scenario is both the most concerning and the most difficult to determine. Any patient who demonstrates objective signs of declining or persistently impaired brain function without a plausible explanation other than exposure to impact forces should be told to avoid further exposure. This evaluation should include a formal neuropsychological evaluation to aid in determining the presence of cognitive decline. As yet, no clear clinical role exists for advanced neuroimaging such as functional MRI, but this and other modalities may be considered. Similarly, the developing fields of quantitative EEG and evoked response potentials may prove useful in the future. If no objective measures show change in brain function or physiology, subjective concerns of dysfunction should also be taken seriously and may warrant the same recommendation, but care should be taken to look for other explanations for this type of concern.

At this time, no clear understanding exists of the risk of developing CTE from chronic exposure to sportsrelated impact forces. Recommendations for retirement can only be made based on clinical experience.

\section{CONCLUSION}

The diagnosis and management of sports concussion is becoming more common in the practice of clinical neurology. While many aspects of sports concussion care should be famil- iar to the practicing neurologist, others require specific consideration. The clinical presentation of concussion has much in common with other diagnoses, and no objective test for injury exists. Therefore, it is often best to describe the degree of certainty of the diagnosis. To that end, appropriate diagnosis and management can only come from applying a comprehensive neurologic approach and understanding the multiple unique factors in play when being asked to provide care for athletes. Concussion diagnosis and management is clearly a clinically based skill that can be significantly improved and honed with experience.

While concern is growing over the potential long-term brain health consequences of concussion and repetitive exposure to biomechanical forces, very little evidence exists that can be used to describe what these risks actually are. Neurologists and other sports health care professionals who seek to provide the best possible care to their athlete-patients must respect the complexities of these issues and must not only consider the negative consequences of brain injury, but also fully understand and appreciate the benefits of playing sports. As science continues to advance in this area, so will our diagnostic approaches and likely our management schema.

\section{REFERENCES}

1. Bogousslavsky J, Tatu L. French neuropsychiatry in the Great War: between moral support and electricity. J Hist Neurosci 2013;22(2):144-154.

2. Denny-Brown DE, Russell WR. Experimental concussion. Proc R Soc Med 1941;34(11): 691-692.

3. Saucier J. Concussion: a misnomer. Can Med Assoc J 1955;72(11):816-820.

4. American Academy of Neurology, Report of the Quality Standards Subcommittee. Practice Parameter: The management of concussion in sports. Neurology 1997;48(3):581-585. 
5. Cantu RC, Gean AD. Second-impact syndrome and a small subdural hematoma: an uncommon catastrophic result of repetitive head injury with a characteristic imaging appearance. J Neurotrauma 2010;27(9):1557-1564.

6. McCrory P, Davis G, Makdissi M. Second impact syndrome or cerebral swelling after sporting head injury. Curr Sports Med Rep 2012;11(1):21-23.

7. Stern RA, Daneshvar DH, Baugh CM, et al. Clinical presentation of chronic traumatic encephalopathy. Neurology 2013;81(13): 1122-1129.

8. Guskiewicz KM, McCrea M, Marshall SW, et al. Cumulative effects associated with recurrent concussion in collegiate football players: The NCAA Concussion Study. JAMA 2003;290(19):2549-2555.

9. McKee AC, Stein TD, Nowinski CJ, et al. The spectrum of disease in chronic traumatic encephalopathy. Brain 2013;136(pt 1):43-64.

10. McCrory P, Meeuwisse WH, Aubry M, et al. Consensus statement on concussion in sport: the 4th International Conference on Concussion in Sport, Zurich, November 2012. Br J Sports Med 2013;47(5):250-258.

11. Harmon KG, Drezner JA, Gammons M, et al. American Medical Society for Sports Medicine position statement: concussion in sport. Br J Sports Med 2013;47(1):15-26.

12. Giza CC, Kutcher JS, Ashwal S, et al. Summary of evidence-based guideline update: evaluation and management of concussion in sports: report of the Guideline Development Subcommittee of the American Academy of Neurology. Neurology 2013;80(24):2250-2257.

13. Sports-related concussions in youth: improving the science, changing the culture. Institute of Medicine of the National Academies website. www.iom.edu/Reports/2013/Sports-RelatedConcussions-in-Youth-Improving-the-ScienceChanging-the-Culture.aspx. Published October 30, 2013. Accessed October 3, 2014.

14. Giza CC, Hovda DA. The neurometabolic cascade of concussion. J Athl Train 2001;36(3): 228-235.
15. Hardman JM, Manoukian A. Pathology of head trauma. Neuroimaging Clin N Am 2002;12(2):175-187.

16. McCrea M, Guskiewicz K, Randolph C, et al. Incidence, clinical course, and predictors of prolonged recovery time following sport-related concussion in high school and college athletes. J Int Neuropsychol Soc 2013;19(1):22-33.

17. Eckner JT, Kutcher JS, Broglio SP, Richardson JK. Effect of sport-related concussion on clinically measured simple reaction time. Br J Sports Med 2014;48(2):112-118.

18. Murray N, Salvatore A, Powell D, Reed-Jones R. Reliability and validity evidence of multiple balance assessments in athletes with a concussion. J Athl Train 2014;49(4):540-549.

19. Galetta KM, Brandes LE, Maki K, et al. The King-Devick test and sports-related concussion: study of a rapid visual screening tool in a collegiate cohort. J Neurol Sci 2011;309(1-2): 34-39.

20. Louey AG, Cromer JA, Schembri AJ, et al. Detecting cognitive impairment after concussion: sensitivity of change from baseline and normative data methods using the CogSport/Axon cognitive test battery. Arch Clin Neuropsychol 2014;29(5):432-441.

21. Tjarks BJ, Dorman JC, Valentine VD, et al. Comparison and utility of King-Devick and ImPACT composite scores in adolescent concussion patients. J Neurol Sci 2013;334(1-2): 148-153.

22. American Academy of Neurology sports concussion toolkit. American Academy of Neurology website. www.aan.com/practice/sportsconcussion-toolkit/. Accessed October 3, 2014.

23. Schneider KJ, Iverson GL, Emery CA, et al The effects of rest and treatment following sport-related concussion: a systematic review of the literature. Br J Sports Med 2013;47(5):304-307.

24. Giza CC, Difiori JP. Pathophysiology of sports-related concussion: an update on basic science and translational research. Sports Health 2011;3(1):46-51. 